\section{Case Reports in Oncology}

\title{
Primary Small Intestinal Sarcomatoid Carcinoma: Report of a Rare Case and Literature Review
}

\author{
Nozomi Karakuchia ${ }^{a}$ Senichiro Yanagawa ${ }^{a}$ Kei Kushitani ${ }^{b}$ \\ Shinya Kodama ${ }^{a}$ Yukio Takeshima $^{\mathrm{b}}$ Kazuo Sumimoto ${ }^{\mathrm{a}, \mathrm{c}}$ \\ aDepartment of Surgery, Yoshida General Hospital, Akitakata, Japan; bepartment of \\ Pathology, Graduate School of Biomedical and Health Sciences, Hiroshima University, \\ Hiroshima, Japan; 'Department of Gastroenterological and Transplant Surgery, Graduate \\ School of Biomedical and Health Sciences, Hiroshima University, Hiroshima, Japan
}

\author{
Keywords \\ Sarcomatoid carcinoma $\cdot$ Small intestine tumor $\cdot$ Surgical resection
}

\begin{abstract}
Sarcomatoid carcinoma (SC) is a rare malignant tumor with properties of both epithelial and mesenchymal carcinomas. SC has been reported in various organs, but the number of reports for each type is small. Small intestinal tumors make up about 3-6\% of gastrointestinal malignancies. Discovering them in the early stage is rare and difficult, with anemia and/or abdominal pain as the major symptoms of small intestinal tumors. Primary small intestinal SC (SISC) is rare among small intestinal tumors, and currently very few cases have been reported in the literature. Previous studies have reported that neither chemotherapy nor radiotherapy improves the overall survival rate of patients with SISC, and the prognosis is extremely poor. Currently, surgical resection remains the only optimal therapeutic approach for SISC. Here, we present the case of a 90-year-old woman who had acute peritonitis due to perforation of a small intestinal tumor. She underwent emergency exploratory laparotomy and partial resection of the small intestine, including the tumor. The tumor was pathologically identified as a primary SISC with mesenteric lymph node metastasis. Subsequently, she had recurrence in the intra-abdominal area and lymph node metastasis anterior to the inferior vena cava and died 15 months after surgery without any additional treatment.




\section{Introduction}

Sarcomatoid carcinoma (SC) is a rare malignant tumor with properties of both epithelial and mesenchymal carcinomas. These tumors have been reported in diverse sites, including the endocrine, respiratory and digestive organs [1].

Primary small intestinal tumors make up approximately 3-6\% of gastrointestinal malignancies [2], among which small intestinal SC (SISC) is rare. Although anemia and/or abdominal pain are the major symptoms of small intestinal tumors, discovering them in an early stage is rare and difficult. Currently, surgical treatment is the optimal therapeutic approach for small intestinal tumors including SISC, as previous studies have reported that neither chemotherapy nor radiotherapy improves the overall survival rate [2, 3].

Here, we report the case of a 90-year-old woman who had acute peritonitis due to perforation of a small intestinal tumor and was pathologically diagnosed as having SISC with lymph node metastasis.

\section{Case Report}

A 90-year-old woman with a past history of hypertension, diabetes, dyslipidemia, and chronic renal failure was admitted to our hospital with abdominal pain. On physical examination, abdominal pain was strong, and rebound tenderness was positive. Laboratory data showed a white blood cell count of 12,000 cells $/ \mu \mathrm{L}, \mathrm{C}$-reactive protein concentration of $41.2 \mathrm{mg} / \mathrm{dL}$, blood urea nitrogen concentration of $38.6 \mathrm{mg} / \mathrm{dL}$, creatinine concentration of $2.13 \mathrm{mg} / \mathrm{dL}$, and an estimated glomerular filtration rate of $17 \mathrm{~mL} / \mathrm{min} / 1.73 \mathrm{~m}^{2}$. An abdominal computed tomography (CT) scan showed free air in the right lower abdominal cavity.

The patient was diagnosed with acute peritonitis due to a gastrointestinal perforation, and exploratory laparotomy was performed. During the operation, a perforation in the small intestine $320 \mathrm{~cm}$ distal to the Treitz ligament on the anal side was identified, and a local abscess had formed between the mesenteries. Partial resection of the small intestine including the tumor and drainage of the abdominal cavity were performed.

Pathological examination revealed the tumor with a maximum diameter of $4 \mathrm{~cm}$ in the small intestine, and the tumor had invaded from the mucosa into the serosa with perforation (Fig. 1a). Histologically, the tumor was characterized by complicated and invasive growth of atypical spindle-shaped cells (Fig. 1b). Immunohistochemically, the tumor cells were broadly positive for cytokeratin-AE1/AE3 (Fig. 1c) and partially positive for $\alpha$-smooth muscle actin and S-100 protein. On the other hand, the tumor cells were negative for desmin, CD34, and c-kit. The Ki-67 labeling index was approximately 50\% in the hot spot (Fig. 1d). In addition, the metastatic tumor was found in one paraintestinal lymph node, and no invasion or metastasis was detected in other organs. The patient was diagnosed with primary SISC of pT4N1M0 pStage IIIA in accordance with the Union for International Cancer Control tumor node metastasis classification 8th edition for small intestinal cancer. After surgery, the patient was discharged on the 18th postoperative day without any complications.

Seven months after the operation, a positron emission tomography (PET)-CT scan showed lymphadenopathy with a maximum standardized uptake value $\left(\mathrm{SUV}_{\max }\right)$ of 7.2 anterior to the inferior vena cava (Fig. 2a) and a tumor with an $S_{\text {Uax }}$ of 8.3 in the abdominal cavity of the right lower abdomen (Fig. 2b). We diagnosed recurrence and metastasis of SISC. The patient and her family refused any treatment because she was asymptomatic at that time.

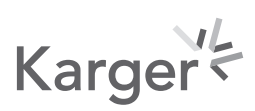




\section{Case Reports in Oncology}
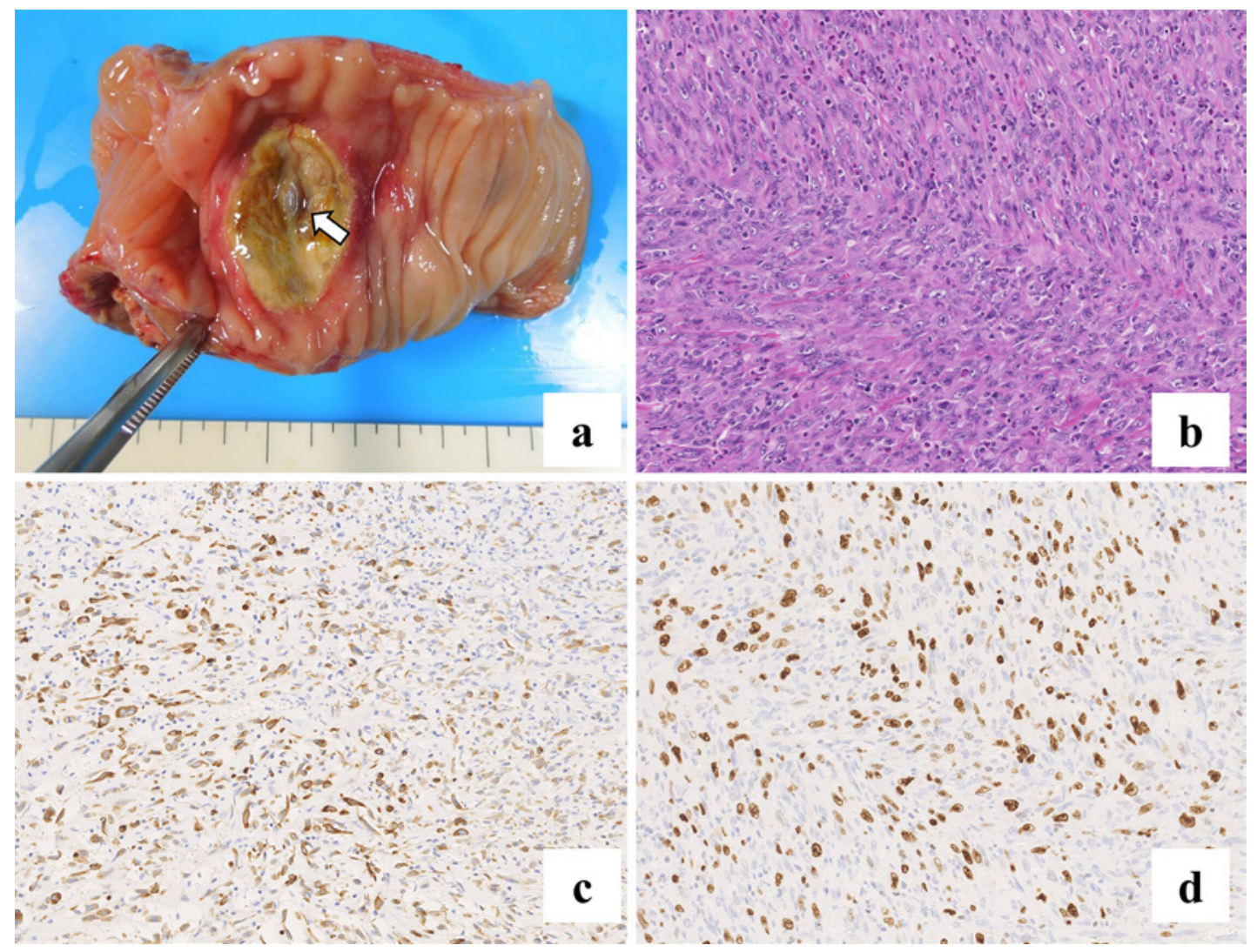

Fig. 1. The tumor is $4 \mathrm{~cm}$ in size with a perforation of $2 \mathrm{~mm}$. The arrow indicates the perforated area (a). $H \& E$ staining shows that the tumor is characterized by spindle-shaped cells in disarray with invasive growth (b). Immunohistochemical findings show that tumor cells are broadly positive for cytokeratin-AE1/AE3 (c), and that the Ki-67 labeling index is about $50 \%$ in the hot spot (d).

The lymph node metastasis had gradually progressed and ascites appeared on CT (Fig. 2c); in addition, the recurrent tumor in the abdominal cavity had rapidly grown to $13 \mathrm{~cm}$ in diameter on the CT scan 7 months after PET-CT imaging (Fig. 2d). The patient died 15 months after the operation.

\section{Discussion and Conclusions}

SC is an extremely rare biphasic tumor characterized by a combination of malignant epithelial and mesenchymal cells. The tumor occurs in various organs, including the digestive, urinary, respiratory and endocrine systems [1]. Although there are case reports of SC in various organs, the number of reports for each type is small.

In general, malignancies of the small intestine are very rare compared with malignancies of other gastrointestinal organs, and they have nonspecific symptoms; thus, early-stage diagnosis is difficult. Adenocarcinoma, neuroendocrine tumors, sarcomas, and lymphomas account for the majority of primary small intestinal tumors [2-4]. Terada [4] reported that the overall 5-year survival rate was 54\% (25\% for adenocarcinoma, 62\% for lymphoma, $83 \%$ for carcinoids, and $45 \%$ for sarcoma), and the Ki-67 labeling index ranged from 40 to $95 \%$ with a mean of $71 \%$ in primary adenocarcinoma, and from 50 to $76 \%$ with a mean of $62 \%$ in 

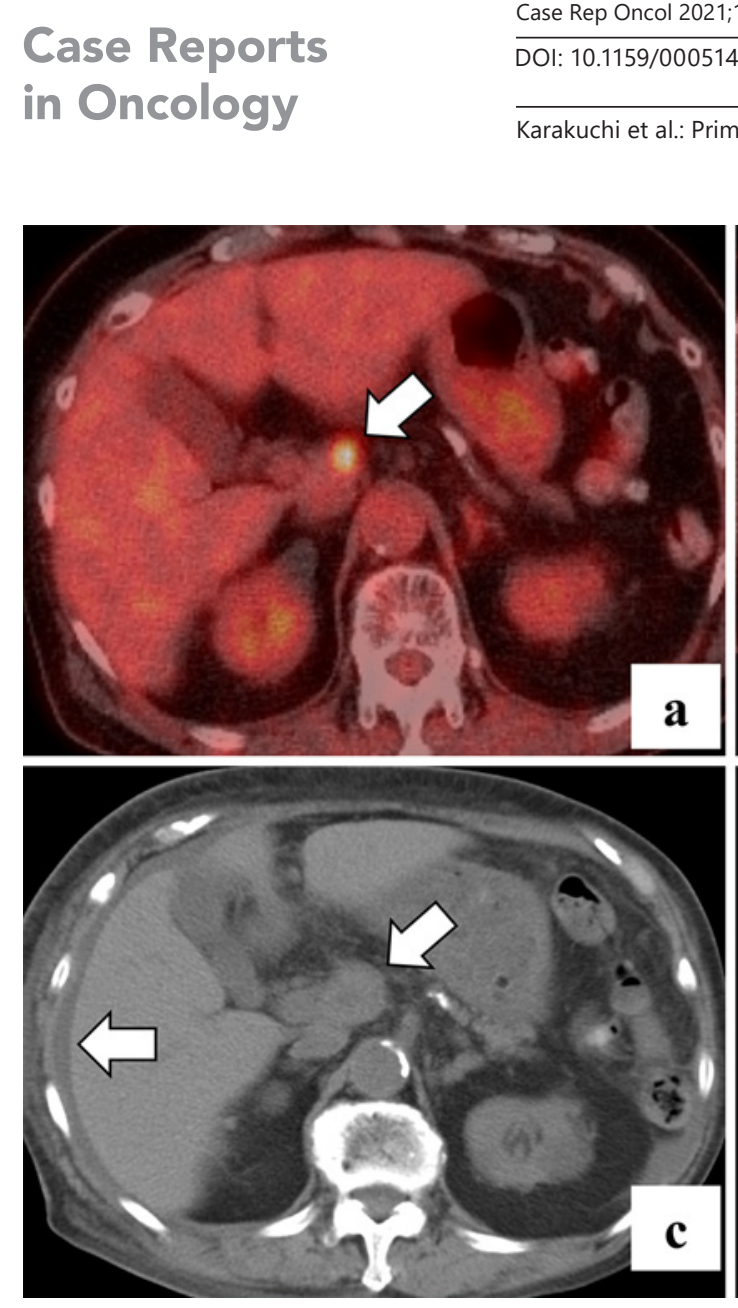

Fig. 2. PET-CT scan at 7 months postoperatively shows lymph node metastasis with an SUV max $_{\text {of }} 7.2$ in front of the inferior vena cava (a; arrow) and recurrence with an $\mathrm{SUV}_{\max }$ of 8.3 in the abdominal cavity of the right lower abdomen (b; arrow). Seven months after the recurrence had been identified, the CT scan shows that ascites appeared (c; arrows), as well as rapid growth of metastatic lymph nodes and the recurrent tumor (c, d; arrows).

squamous cell carcinoma. These data suggest active proliferation in small intestinal carcinoma. Risk factors for small intestinal tumors include celiac disease, inflammatory bowel disease, and a number of genetic abnormalities [3]; it is unclear whether these risk factors apply to SISC, but the prognosis of SISC is extremely poor [5].

The clinical manifestations of SISC are remarkably nonspecific, including abdominal pain, bowel obstruction, a palpable abdominal mass, nausea, vomiting, weight loss, gastrointestinal bleeding, and anemia. These symptoms always occur when the cancer has reached an advanced stage.

In our review of the literature, 9 cases of primary SISC have been reported in the last decade [6-14], and these 10 cases (including the present case) are shown in Table 1. Abdominal pain was noted in 7 cases $[6-9,13,14]$, and 3 cases, including the present case, were complicated by peritonitis due to tumor perforation $[6,9]$.

Since a diagnosis by hematoxylin and eosin (H\&E) staining alone is insufficient, immunohistochemical diagnosis, such as with vimentin or cytokeratin (e.g., AE1/AE3, CAM5.2), is necessary. These tumors are extremely fast-growing, and patients have a survival of less than 1 year postoperatively [6,10-13]. At diagnosis, more than half of patients had large tumors ( $\geq 5 \mathrm{~cm}$ ) with widely spread local disease (Table 1 ); SISC is a highly invasive tumor. 
Case Reports

in Oncology
Case Rep Oncol 2021;14:538-544 DOI: $10.1159 / 000514145$

(c) 2021 The Author(s). Published by S. Karger AG, Basel www.karger.com/cro

Karakuchi et al.: Primary Small Intestinal Sarcomatoid Carcinoma

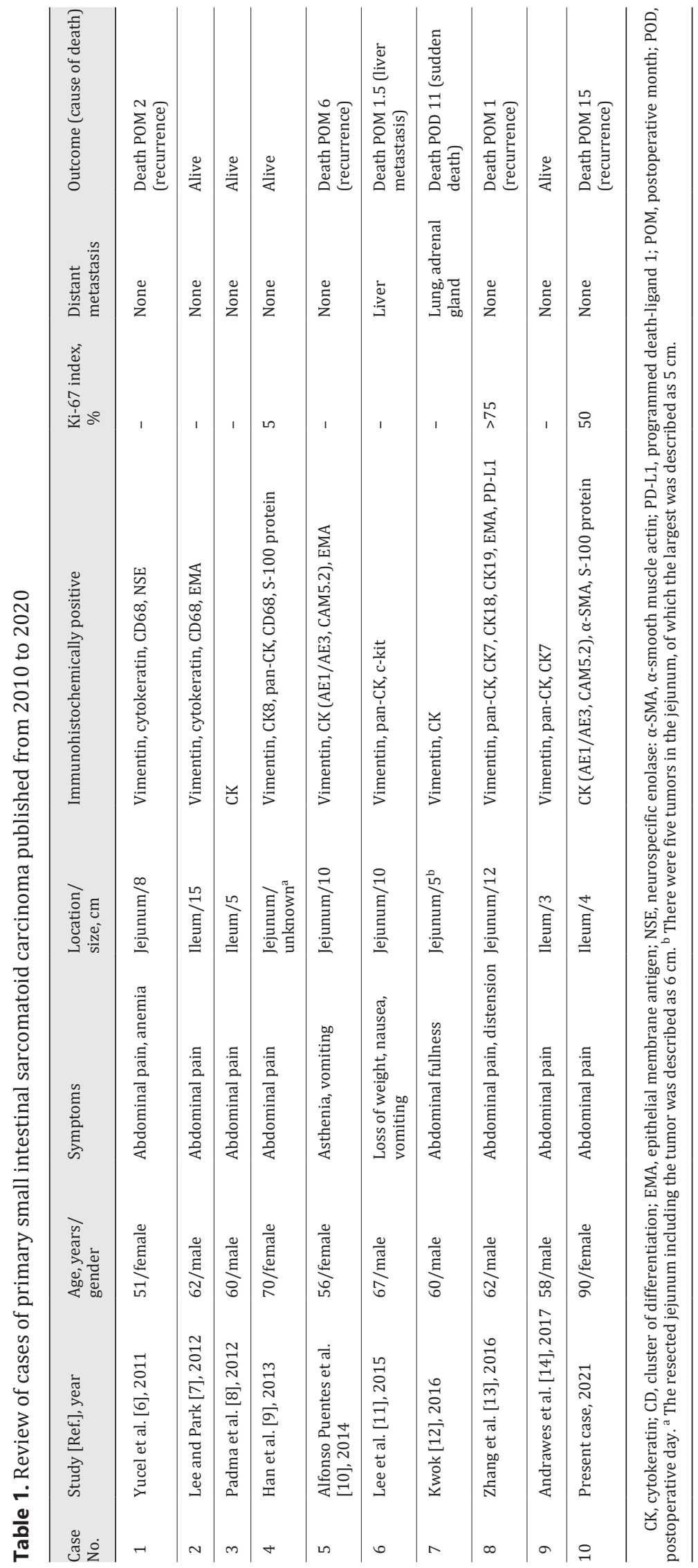

Karger's 
There is currently no consensus regarding the treatment for SISC. The first choice of treatment for SISC is surgical resection, but the disease often recurs after surgery. In addition, there have been reports of chemotherapy administration (e.g., doxorubicin, dacarbazine, ifosfamide, gemcitabine, docetaxel, taxane, and platinum) for SISC and SC of unknown primary origin, but none of them were as effective as expected [1, 5, 7]. Lee and Park [7] reported that they performed surgical resection for a pelvic recurrence of SISC and restarted chemotherapy. Since there are no case reports of effective chemotherapy, aggressive surgical resection may be effective against recurrence of SISC. Regarding prognosis, 6 of the 10 patients died, and 5 died within 6 months postoperatively (Table 1). All 4 surviving cases were reported within 1 year after surgery; thus, survival of 1 year or longer is unknown.

In the present case, the patient underwent partial resection of the small intestine including the tumor with intraperitoneal lavage, and she was pathologically diagnosed with primary SISC using the excised specimen. The Ki-67 index had a value of $50 \%$ in the hot spot, and the cell proliferation ability was high. Luo et al. [15] reported a correlation between Ki-67 incidence and prognosis in gastrointestinal cancers; therefore, we classified this SISC as highly malignant. This case was predicted to have a poor prognosis; however, she was able to survive for 15 months postoperatively, which suggests that surgery could have prolonged her survival time. In addition, the prognosis might have improved slightly if aggressive surgery had been performed when the PET-CT image showed two areas of recurrence after the operation.

In conclusion, aggressive surgical resection for SISC may improve the prognosis, if the patient's condition allows it.

\section{Acknowledgements}

The authors would like to thank the patient's family for providing consent to publish the clinical information and data in this report. We would like to thank Editage (www.editage. com) for English language editing.

\section{Statement of Ethics}

Written informed consent was obtained from the patient's son for publication of this case report and any accompanying images.

\section{Conflict of Interest Statement}

The authors have no conflicts of interest to declare.

\section{Funding Sources}

The authors received no financial support for the research, authorship, or publication of this article.

\section{Karger'}




\section{Case Reports in Oncology}

Case Rep Oncol 2021;14:538-544

\begin{tabular}{l|c|c|}
\hline DOI: $10.1159 / 000514145$ & $\odot 2021$ The Author(s). Published by S. Karger AG, Basel
\end{tabular} www.karger.com/cro

Karakuchi et al.: Primary Small Intestinal Sarcomatoid Carcinoma

\section{Author Contributions}

N.K. and S.Y. made a substantial contributions to the concept and design of the report as well as to data acquisition and interpretation. K.K., S.K., Y.T., and K.S. were involved in drafting the manuscript and critical revision of the intellectual content. S.Y. approved the final version of the manuscript submitted for publication. All authors have read and approved this manuscript.

\section{References}

1 Huey RW, Makawita S, Xiao L, Matamoros A, Estrella JS, Overman MJ, et al. Sarcomatoid carcinoma presenting as cancers of unknown primary: a clinicopathological portrait. BMC Cancer. 2019 Oct;19(1):965.

2 Taghipour Zahir S, Heidarymeybodi Z, AleSaeidi S. Prognostic factors and survival time in patients with small bowel tumors: a retrospective observational study. Int J Surg Oncol. 2019 May;2019:2912361.

3 Reynolds I, Healy P, Mcnamara DA. Malignant tumours of the small intestine. Surgeon. 2014 Oct;12(5):26370.

4 Terada T. Malignant tumors of the small intestine: a histopathologic study of 41 cases among 1,312 consecutive specimens of small intestine. Int J Clin Exp Pathol. 2012;5(3):203-9.

5 Reid-Nicholson M, Idrees M, Perino G, Hytiroglou P. Sarcomatoid carcinoma of the small intestine: a case report and review of the literature. Arch Pathol Lab Med. 2004 Aug;128(8):918-21.

6 Yucel AF, Kocakusak A, Arikan S, Demirbag N, Tarlaci A, Batur S. A rare cause of acute abdomen: perforated primary sarcomatoid carcinoma of the small intestine - report of a case, with a brief review of the literature. J Cancer Res Ther. 2011 Jul; 7(3):348-50.

7 Lee SE, Park SY. Sarcomatoid carcinoma of the small intestine: a rare and highly aggressive tumor. J Korean Surg Soc. 2012 Nov;83(5):321-4.

8 Padma SK, Permi HS, Patil CN, Mathias M. Aggressive monophasic sarcomatoid carcinoma of small intestine - a rare case report with review of literature. Nitte Univ J Health Sci. 2012;2(1):45-7.

9 Han N, Han QH, Liu YZ, Li ZC, Li J. Perforated sarcomatoid carcinoma of the jejunum: case report. Oncol Lett. 2013 Aug;6(2):562-4.

10 Alfonso Puentes N, Jimenez-Alfaro Larrazabal C, García Higuera MI. Sarcomatoid carcinoma of the jejunum presenting as obscure gastrointestinal bleeding in a patient with a history of gliosarcoma. Gastroenterol Rep (Oxf). 2014;2(2):150-3.

11 Lee HM, Cho MS, Kim YI. A surgically resected large sarcomatoid carcinoma of the jejunum: a case report and literature review. J Gastric Cancer. 2015 Jun;15(2):143-6.

12 Kwok CM. Sarcomatoid carcinoma of the jejunum with gastric metastases: a case report and review of the literature. Int J Surg Case Rep. 2016;28:161-4.

13 Zhang B, Cheng BO, Wang L, Zhao KE, Zhuo GZ, Ding JH. Primary sarcomatoid carcinoma of the jejunum with massive intra-abdominal hemorrhage: a case report and review of the literature. Mol Clin Oncol. 2016 May; 4(5):811-6.

14 Andrawes PA, Shariff M, Chang Q, Grinberg R. Primary sarcomatoid carcinoma of the small intestine: very rare and aggressive tumour. BMJ Case Rep. 2017 Jan;2017:bcr2016217895.

15 Luo Z-W, Zhu M-G, Zhang Z-Q, Ye F-J, Huang W-H, Luo X-Z. Increased expression of Ki-67 is a poor prognostic marker for colorectal cancer patients: a meta analysis. BMC Cancer. 2019 Feb;19(1):123. 